\title{
Exposição dos Doentes Internados nas Unidades José de Mello Saúde a Antibacterianos
}

\section{Exposure of Inpatients to Antibacterials at the José de Mello Saúde Hospital Units}

Ana Freitas ${ }^{1}$, Ana Plácido², Ana Vinagre ${ }^{3}$, Carla Ferrer ${ }^{4}$, Joana Cardoso ${ }^{5}$, Joana Guerra ${ }^{5}$, Joana Marques ${ }^{6}$, José Branco ${ }^{4}$, Maria Pereira 7 , Pedro Almeida ${ }^{3}$, Rita Oliveira ${ }^{8}$, Sara Barroso ${ }^{9}$, Sílvia Martins ${ }^{6}$

\section{RESUMO}

INTRODUÇÃO: O uso racional dos antibacterianos é um fator crítico no que concerne ao controlo da emergência da resistência a antimicrobianos, constituindo uma problemática global de saúde pública. Assim, a José de Mello Saúde considerou relevante efetuar um estudo retrospetivo do uso de medicamentos nos anos 2014 e 2015 com o objetivo de efetuar uma análise comparativa do consumo de antimicrobianos intra e inter unidades, de modo a contribuir para o uso racional dos antimicrobianos e a otimizar a política de prevenção e controlo de infeções nas diferentes unidades hospitalares da José de Mello Saúde.

MATERIAL E MÉTODOS: Foi analisado o consumo de antimicrobianos por código ATC (anatomical therapeutic chemical), expresso em dose diária definida (DDD) por 100 camas ocupadas/dia, em seis Unidades Hospitalares: CUF Infante Santo, CUF Descobertas, CUF Cascais, CUF Porto, Hospital de Vila Franca de Xira e Hospital de Braga.

DISCUSSÃO: Os resultados obtidos mostram que no período estudado houve um aumento do consumo de antibióticos e que se registou um consumo preponderante de antibióticos por via parentérica, o que demonstra a necessidade de implementação de políticas de utilização racional desta classe de medicamentos, bem como a uniformização de boas práticas e a utilização de protocolos de uso/prescrição de antimicrobianos transversal a todas as unidades.

PALAVRAS-CHAVE: Antibacterianos; Doentes Internados; Farmacorresistência Bacteriana; Revisão de Uso de Medicamentos

\section{ABSTRACT}

INTRODUCTION: The rational use of antibacterials is a critical factor in controlling the emergence of antimicrobial resistance, and represents a global public health issue. Thus, José de Mello Saúde considered it relevant and developed a retrospective study about antibacterials utilization in the years 2014 and 2015 by a comparative analysis of the use of antimicrobials intra

1. Serviços Farmacêuticos Hospital CUF Descobertas; Farmácia Cluster Descobertas. 2. Serviços Farmacêuticos Hospital de Braga; Farmácia Cluster Norte. 3. Serviços Farmacêuticos Hospital CUF Porto. 4. Serviços Farmacêuticos Hospital de Vila Franca de Xira. 5. Serviços Farmacêuticos Hospital CUF Infante Santo. 6. Serviços Farmacêuticos Hospital CUF Descobertas. 7. Serviços Farmacêuticos José de Mello Saúde. 8. Serviços Farmacêuticos Hospital CUF Infante Santo; Farmácia Cluster Tejo. 9. Serviços Farmacêuticos Hospital de Braga.

Recebido: 20/04/2017 - Aceite: 12/05/2017 
and interunits, setting as the main goal the rational use of antimicrobials and the optimization of prevention and control of infections policies in the different hospital units of José de Mello Saúde.

MATERIALS AND METHODS: Antibacterial utilization was analysed by ATC (anatomical therapeutic chemical) code expressed in daily defined dose (DDD) per 100 beds per day was analyzed in six Hospital Units: CUF Infante Santo, CUF Descobertas, CUF Cascais, CUF Porto, Hospital de Vila Franca de Xira and Hospital de Braga.

DISCUSSION: During the study period, there was an increase in the overall use of antibacterials and a preponderant utilization of antibacterials by parenteral route, which demonstrates the need to implement policies that can promote the rational use of this class of drugs, as well as the standardization of good practices and the use of antibacterials use and or prescription protocols across all units.

KEYWORDS: Antibacterial Agents; Drug Resistance, Bacterial; Drug Utilization Review; Inpatients

\section{INTRODUÇÃO}

Atualmente, o uso racional dos antibacterianos é um fator crítico no que concerne à emergência da resistência aos antimicrobianos, constituindo uma problemática global de saúde pública. ${ }^{1}$ É um facto que as estirpes microbianas multirresistentes têm vindo a aumentar em consequência do uso incorreto de antimicrobianos, principalmente devido à prescrição indiscriminada e/ou falha de cumprimento dos regimes terapêuticos. ${ }^{2}$

Deste modo, é crucial a monitorização do consumo de antibacterianos em áreas que vão desde os estudos clínicos aos farmacoepidemiológicos e microbiológicos. ${ }^{3}$ Para a avaliação do consumo de antimicrobianos é recomendado pela Organização Mundial de Saúde (OMS) utilizar a metodologia código ATC (anatomic therapeutic chemical)/DDD (defined daily dose). ${ }^{4}$ A DDD é definida como a dose média de manutenção por dia de um medicamento para a sua principal indicação em adultos. ${ }^{4}$ No entanto, é importante salientar que esta unidade de medida não reflete necessariamente a dose diária recomendada de prescrição, sendo apenas um indicador de estimativa de consumo. ${ }^{4}$

Segundo dados do ECDC (European Centre for Disease Prevention and Control), em 2014 o consumo médio de antibacterianos de uso sistémico (ATC J01) no setor hospitalar, em Portugal (1,55/1000 habitantes/dia), foi inferior à média europeia (2,06/1000 habitantes/dia). ${ }^{5}$ No que respeita apenas à realidade em Portugal, segundo dados desta mesma entidade, o consumo de antimicrobianos sistémicos tem vindo a aumentar desde 2009 a 2014 (de 1,41 a 1,55/1000 habitantes/dia).6,7

Face a este problema, têm sido implementadas algumas iniciativas a nível global. Em Portugal, o Ministério da Saúde emitiu o despacho n 2902/2013, o qual determinou a criação do Programa de Prevenção e Controlo de Infeção e Resistência aos Antimicrobianos (PPCIRA) de modo a abranger as três vertentes essenciais de um programa global de prevenção e controlo de infeção, como sejam a vigilância epidemiológica, a elaboração e a monitorização do cumprimento de normas e recomendações de boa prática, bem como a formação dos profissionais de saúde (DGS). ${ }^{8}$ Em 2016, foi emitido o Despacho n. ${ }^{\circ}$ 3844-A/2016 em Diário da República, que refere indicadores de qualidade PPCIRA. ${ }^{9}$

De modo a que a prevenção e controlo das infeções associadas aos cuidados de saúde (IACS) seja vista como parte integrante das atividades diárias dos profissionais, contribuindo para a qualidade dos cuidados e para a segurança dos doentes, a José de Mello Saúde teve necessidade de adquirir uma ferramenta de trabalho, o HEPIC (Hospital Epidemiologic Control). Esta ferramenta tem como principais funcionalidades a integração automática de resultados laboratoriais, a avaliação do consumo de antimicrobianos, a monitorização de multirresistências, eventos sentinela e deteção de surtos, e ainda permite estudos de epidemiologia no Hospital. ${ }^{10}$

Tendo em conta a importância desta problemática, a José de Mello Saúde (JMS) considerou relevante fazer um estudo retrospetivo dos anos 2014 e 2015, no qual se avaliou o consumo de antimicrobianos por código ATC expresso em DDD por 100 camas ocupadas, em seis Unidades Hospitalares: CUF Infante Santo, CUF Descobertas, CUF Cascais, CUF Porto, Hospital de Vila Franca de Xira e Hospital de Braga. Em suma, o principal objetivo deste estudo foi comparar o consumo de antimicrobianos intra e inter unidades e, consequentemente, contribuir para o uso racional dos antimicrobianos e otimizar a política de prevenção e controlo de infeções nas diversas unidades hospitalares José de Mello Saúde.

O estudo tem como objetivo principal contribuir para o uso racional dos antimicrobianos nas unidades JMS. São objetivos específicos analisar o consumo de antimicrobianos entre os anos 2014 e 2015 por classe terapêutica, verificar a proporção do consumo anual de 
antimicrobianos de uso parentérico e oral e efetuar uma análise comparativa do consumo de antimicrobianos nas diferentes unidades JMS.

\section{MATERIAL E MÉTODOS}

Análise retrospetiva de dados de consumo de antimicrobianos durante os anos de 2014 e 2015, de forma a proceder ao cálculo das DDD/100 internamentos/dia, referentes aos doentes internados nas unidades do grupo José de Mello (Hospital de Vila Franca de Xira, Hospital CUF Descobertas, Hospital CUF Infante Santo, Clínica CUF Cascais, Hospital de Braga e Hospital CUF Porto).

\section{Consumos de Antibacterianos em DDD/100} Internamentos/dia

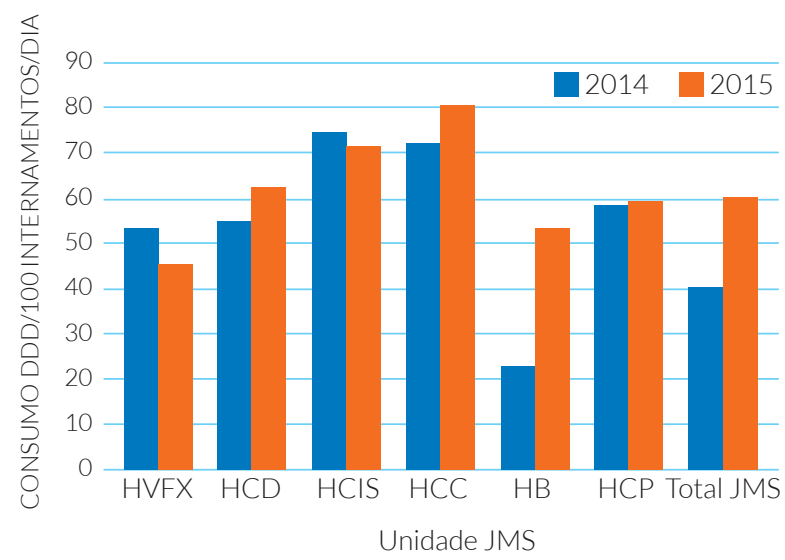

FIGURA 1. Evolução anual do consumo total de antimicrobianos nas diferentes unidades da JMS.
Os dados de consumo de antimicrobianos foram retirados da plataforma informática GLINTT, sendo os valores de internamentos/dia retirados da plataforma informática SIG.

Neste estudo assume-se que a prescrição de antimicrobianos corresponde ao seu consumo, expresso em DDD/100 doentes internados/dia (DDD).

\section{A fórmula aplicada foi:}

DDD $=$ ( $n^{\circ}$ de DDDs consumidas $/\left(n^{\circ}\right.$ de internamentos $/$ dia)) $\times 100$

O cálculo do número de doses diárias definidas consumidas baseia-se na fórmula:

( $\mathrm{N}^{\circ}$ de unidades consumidas $\times$ dosagem de cada unidade)/DDD de cada antibiótico

A DDD atribuída a cada antibiótico para efeitos de cálculo da DDD consumida, assim como a classificação das substâncias ativas, baseiam-se no ATC Index 2016 da OMS, independentemente do ano a que se refere o consumo.

\section{RESULTADOS}

A análise dos dados obtidos (Fig. 1) mostra que a unidade com maior consumo de antibacterianos em DDD por 100 internamentos/dia, em 2014 foi o Hospital CUF Infante Santo e em 2015 o Hospital CUF Cascais.

No período em análise verificou-se um aumento de consumo em todas as unidades, exceto o HVFX e HCIS que apresentaram um menor consumo em 2015 face a 2014.

TABELA 1. Consumo de antibacterianos (DDD por 100 internamentos/dia) no ano de 2014 por classe farmacoterapêutica.

\begin{tabular}{|l|c|c|c|c|c|c|c|}
\cline { 2 - 8 } & \multicolumn{9}{c|}{} & \multicolumn{2}{c|}{2014} & HB & HCP \\
\cline { 2 - 8 } & HVFX & HCD & HCIS & HCC & 23,68 & 5,32 & 27,24 \\
\hline Cefalosporinas & 7,45 & 12,66 & 22,53 & 4,13 & 1,96 & 2,6 \\
\hline Quinolonas & 3,11 & 4,72 & 4,06 & 7,89 & 1,96 & 1,01 \\
\hline Macrólidos & 6,58 & 6,86 & 14,45 & 5,64 & 1,31 & 1,7 \\
\hline Penicilinas & 4,34 & 6,66 & 5,75 & 20,24 & 8,48 & 15,07 \\
\hline Outros antibacterianos & 23,58 & 13,76 & 16,88 & 11,2 & 4,07 & 5,5 \\
\hline
\end{tabular}

TABELA 2. Consumo de antibacterianos (DDD por 100 internamentos/dia) no ano de 2015 por classe farmacoterapêutica.

\begin{tabular}{|c|c|c|c|c|c|c|}
\hline & \multicolumn{6}{|c|}{2015} \\
\hline & HVFX & HCD & $\mathrm{HCIS}$ & $\mathrm{HCC}$ & $\mathrm{HB}$ & $\mathrm{HCP}$ \\
\hline Cefalosporinas & 7,8 & 19,21 & 19,57 & 27,31 & 12 & 28,52 \\
\hline Carbapenemes & 3,21 & 4,84 & 5,62 & 6,24 & 5,04 & 2,94 \\
\hline Quinolonas & 4,48 & 6,07 & 13,7 & 7,97 & 4,5 & 6,54 \\
\hline Macrólidos & 3,91 & 5,63 & 5,87 & 6,56 & 3,24 & 1,89 \\
\hline Penicilinas & 20,11 & 15,5 & 16,25 & 21,08 & 18,51 & 13,93 \\
\hline Outros antibacterianos & 6,08 & 10,96 & 11,2 & 11,65 & 9,89 & 5,48 \\
\hline
\end{tabular}


Distribuição do consumo de antibacterianos por classe farmacoterapêutica (2014)

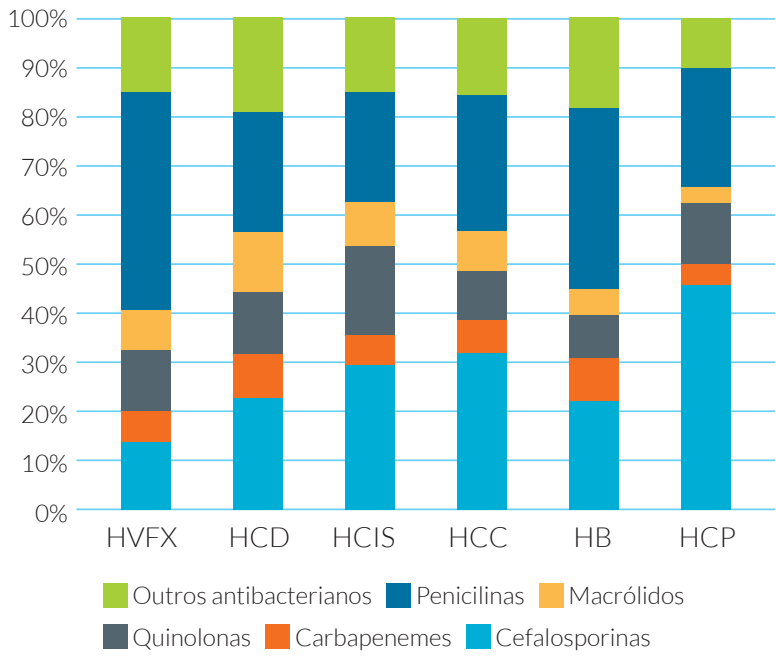

FIGURA 2. Distribuição do consumo de antibacterianos no ano de 2014 por classe farmacoterapêutica.

Em 2014 (Tabela 1 e Fig. 2) a classe de antimicrobianos mais consumida nas unidades HVFX, HCD e HB foi a das penicilinas, ao contrário de HCIS, HCC e HCP em que a classe com maior consumo foi a das cefalosporinas.

As classes penicilinas e cefalosporinas foram as duas em que se verificou o consumo mais elevado em todas as unidades, com exceção do HVFX, em que a $2^{\text {a }}$ classe mais consumida foi a de outros antibacterianos.

A terceira classe mais consumida nas unidades HCD, HCC e HB foi a classe outros antibacterianos. No HVFX a terceira classe mais consumida foi a das cefalosporinas, enquanto no HCIS e no HCP esta correspondeu à classe das quinolonas.

Em 2015 (Fig. 3 e Tabela 2), as duas classes de antibacterianos com maior consumo foram novamente penicilinas e cefalosporinas, em todas as unidades do grupo.
Distribuição do consumo de antibacterianos por classe farmacoterapêutica (2015)

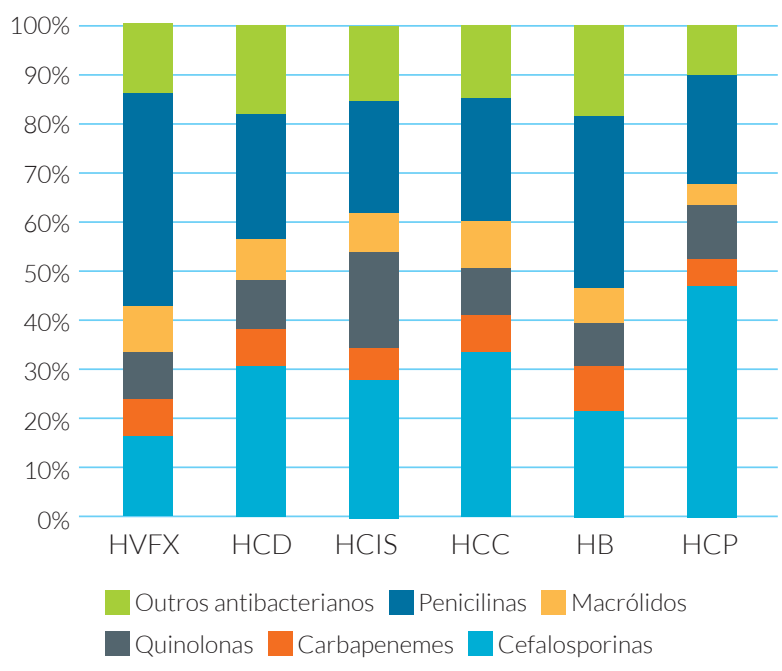

FIGURA 3. Distribuição, em percentagem, do consumo de antibacterianos no ano de 2015 por classe farmacoterapêutica.

A classe com maior consumo nas unidades $\mathrm{HCD}, \mathrm{HCIS}$, HCC e HCP foi a classe das cefalosporinas, enquanto no HVFXe HB foi a classe das penicilinas.

A terceira classe com maior consumo manteve-se em cada unidade, relativamente a 2014, com exceção do HVFX, em que esta passou a ser a classe dos outros antibacterianos.

Em todas as unidades JMS o consumo de carbapenemes aumentou em 2015 relativamente a 2014.

$\mathrm{Na}$ classe das quinolonas verificou-se uma diminuição do consumo em todas as unidades, com exceção do HCC e HB.

A análise da evolução do consumo de antibacterianos por via parentérica versus via oral de 2014 para 2015 por unidade JMS (Tabela 3, Tabela 4 e Fig. 4) mostrou que relativamente à via de administração de antibacterianos, de 2014 para 2015 verificou-se um aumento da

TABELA 3. Percentagem de consumo de antibacterianos por via parentérica versus por via oral no ano de 2014 nas diferentes unidades da JMS.

\begin{tabular}{|c|c|c|c|c|c|c|}
\hline & \multicolumn{6}{|c|}{2014} \\
\hline & HVFX & HCD & $\mathrm{HClS}$ & $\mathrm{HCC}$ & HB & HCP \\
\hline Parentérica & $78,49 \%$ & $71,67 \%$ & $70,83 \%$ & $75,49 \%$ & $84,60 \%$ & $84,20 \%$ \\
\hline Oral & $21,51 \%$ & $28,33 \%$ & $29,17 \%$ & $24,51 \%$ & $15,40 \%$ & $15,80 \%$ \\
\hline
\end{tabular}

TABELA 4. Percentagem de consumo de antibacterianos por via parentérica versus por via oral no ano de 2015 nas diferentes unidades da JMS.

\begin{tabular}{|l|c|c|c|c|c|c|}
\hline \multirow{2}{*}{} & \multicolumn{7}{c|}{2015} \\
\cline { 2 - 7 } & HVFX & HCD & HCIS & HCC & HB & HCP \\
\hline Parentérica & $79,07 \%$ & $76,92 \%$ & $67,00 \%$ & $81,43 \%$ & $83,27 \%$ & $87,45 \%$ \\
\hline Oral & $20,93 \%$ & $23,08 \%$ & $33,00 \%$ & $18,57 \%$ & $16,73 \%$ & $12,55 \%$ \\
\hline
\end{tabular}




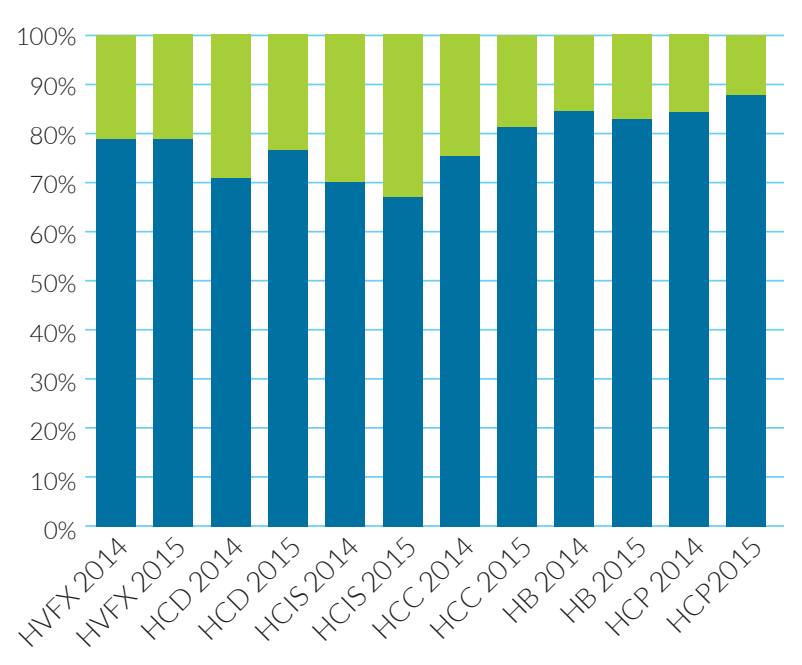

Oral

Parentérica

FIGURA 4. Distribuição do consumo de antibacterianos por via parentérica versus por via oral nos anos de 2014 e 2015 nas diferentes unidades da JMS.

percentagem de antibacterianos administrados por via parentérica em todas as unidades JMS, excetuando o HCIS e HB em que o aumento se verificou na percentagem de antibacterianos administrados por via oral.

É de salientar o facto de que em todas as unidades, no período estudado, a percentagem de antimicrobianos administrados por via parentérica foi superior a 70\%, excetuando no HCIS para o ano de 2015. O valor mais alto de percentagem de antimicrobianos administrados por via parentérica registou-se no HCP em 2015, atingindo o valor de $87,45 \%$ das DDDs de antimicrobianos administrados.

\section{DISCUSSÃO}

O estudo teve como limitação a exclusão do Serviço de Urgência e Bloco Operatório pela inexistência de registos fidedignos que permitam a imputação do consumo dos antibióticos ao doente.

Pela análise dos resultados obtidos no período 2014 e 2015, concluiu-se que houve um aumento da exposição a antimicrobianos nos doentes internados nas unidades JMS.

Através dos dados obtidos sobre a variação do consumo de antimicrobianos por via parentérica versus oral, concluiu-se que há um consumo elevado por via parentérica. A criação de um protocolo de "switch" IV para oral, poderia contribuir para assegurar que o mesmo seja efetuado devidamente, diminuindo o custo e as desvantagens relacionados com uma administração parentérica desnecessária.

Uma vez que se verificou um aumento no consumo de carbapenemes em todas as unidades JMS, uma políti- ca transversal a todo o grupo poderia contribuir para o cumprimento das metas definidas no despacho 3844A/2016, nomeadamente a redução de 10\% ao ano no consumo de carbapenemes.

\section{CONCLUSÃO}

Os dados obtidos mostraram que os objetivos específicos foram atingidos e que, apesar de se tratar de um estudo retrospetivo, revelaram-se pertinentes na definição de implementação de medidas de racionalização do uso dos medicamentos alvo deste estudo.

A caracterização da utilização de antibacterianos nas diferentes unidades e a análise comparativa permitiu perceber que, ainda que representem realidades distintas, é fundamental a implementação de uma política que garanta o uso racional de antimicrobianos, a uniformização de boas práticas e a utilização de protocolos de uso/prescrição de antimicrobianos, transversal a todas as unidades.

A monitorização da implementação destas medidas e a constante redefinição de estratégias permitirá, por sua vez, contribuir para o uso racional dos antimicrobianos em todas as unidades da JMS.

CONFLITOS DE INTERESSE: Os autores declaram não ter qualquer conflito de interesse na realização do presente trabalho.

FONTES DE FINANCIAMENTO: Não houve qualquer fonte de financiamento na realização do presente trabalho.

CONFIDENCIALIDADE DOS DADOS: Os autores declaram ter seguido os protocolos da sua instituição acerca da publicação dos dados de doentes.

PROTEÇÃO DE PESSOAS E ANIMAIS: Os autores declaram que os procedimentos seguidos na elaboração do presente trabalho estão em conformidade com as normas das comissões de investigação clínica e de ética, bem como da declaração de Helsínquia e da Associação Médica Mundial.

CONFLICTS OF INTEREST: The authors declare that they have no conflicts of interest.

FINANCIAL SUPPORT: This work has not received any contribution, grant or scholarship.

CONFIDENTIALITY OF DATA: The authors declare that they have followed the protocols of their work center on the publication of data from patients.

PROTECTION OF HUMAN AND ANIMAL SUBJECTS: The authors declare that the procedures followed were in accordance with the regulations of the relevant clinical research ethics committee and with those of the Code of Ethics of the World Medical Association (Declaration of Helsinki). 


\section{REFERÊNCIAS}

1. Garcell HG, Arias AV, Fernandez EA, Guerrero YB, Serrano RN. Antibiotic Consumption during a 4-year period in a community hospital with an antimicrobial stewardship program. Oman Med J. 2016;31:352-6.

2. Oza A, Donohue F, Johnson H, Cunney R. Risk-adjusted antibiotic consumption in 34 public acute hospitals in Ireland, 2006 to 2014. Euro Surveill. 2016;21(32).

3. Caldeira L, Teixeira I, Vieira I, Batel Marques F, Santiago LM, Rodrigues $\mathrm{V}$, et al. Monitorização do consumo de antibióticos nos Serviços de Cirurgia e de Ortopedia de Seis Hospitais SA. Acta Med Port 2006;19:55-66.

4. World Health Organization (WHO). ATC/DDD Index 2016. Last updated: 2015-12-16. [accessed Dec 2016] Available from: http://www.whocc.no/atc_ddd_index/.

5. European Centre for Disease Prevention and Control. consumption (ECDC). Summary of the latest data on antibiotic consumption in the European Union. 2015. [accessed Dec 2016] Available from: http://ecdc.europa.eu/en/eaad/antibiotics-get-informed/antibiotics-resistance-consumption/pages/ data-reports.aspx.

6. European Centre for Disease Prevention and Control. consumption (ECDC). Antimicrobial consumption rates by country. Last update: 2016-10-17. [accessed Dec 2016] Available from: http://ecdc.europa.eu/en/healthtopics/antimicrobial-resistance-and-consumption/antimicrobial-consumption/esac-net-database/Pages/Antimicrobial-consumption-rates-by-country. aspx.
7. European Centre for Disease Prevention and Control. consumption (ECDC). Trend of the consumption of antibacterials for systemic use (ATC group J01) in the hospital sector in Portugal from 1997 to 2014. Last update: 2016-10-17. [accessed Dec 2016] Available from: http://ecdc.europa.eu/ en/healthtopics/antimicrobial-resistance-and-consumption/ antimicrobial-consumption/esac-net-database/Pages/trend-consumption-by-country.aspx.

8. Ministério da Saúde do Governo de Portugal. Despacho no 2902/2013: Programa de Prevenção e Controlo de Infeção e Resistência aos Antimicrobianos (PPCIRA). [accessed Dec 2016] Available from: http://www.arsalgarve.min-saude.pt/ portal/sites/default/files//images/centrodocs/normas/normas_IACS/Dspcho_2902_2013_MS.pdf.

9. Ministério da Saúde do Governo de Portugal. Despacho n. 3844-A/2016: Diário da República, 2. série - N. 52 - 15 de março de 2016.

10. First solution. Manual de utilizador HEPIC v3. 2009. [accessed Dec 2016] Available from: http://www.first-global.com/pt-pt/ Solucoes/Hepic. 